\title{
DAMPING WIGGLER STUDY AT KEK-ATF
}

\author{
T.Naito, H.Hayano, Y.Honda, K.Kubo, M.Kuriki, S.Kuroda, T.Muto, N.Terunuma, J.Urakawa, \\ KEK(High Energy Accelerator Research Organization), Tsukuba, Japan \\ H.Sakai, N.Nakamura, ISSP, Univ. of Tokyo, Kashiwa, Japan \\ M.Korostelev, F.Zimmermann, CERN, Geneva, Switzerland \\ Marc Ross, SLAC, Menlo Park, California
}

\begin{abstract}
The effects of damping wiggler magnets have been studied at KEK-ATF damping ring, which is a $1.3 \mathrm{GeV}$ storage ring capable of producing ultra-low emittance electron beams. The fast beam damping is a significant issue for the damping ring. The tuning method with 4 sets of wiggler magnets was investigated for the ultra-low emittance beam. The effect on the beam quality, which is related to the transverse ( $\mathrm{x}$ and $\mathrm{y}$ ) and the longitudinal $(\mathrm{z}$ and $\Delta \mathrm{p} / \mathrm{p})$, has been measured by the wire scanner, SR monitor, the laser wire, streak camera and the energy spread monitor. We report on the operational condition and the measurement results.
\end{abstract}

\section{INTRODUCTION}

The performance of the damping wiggler magnet is a significant issues for the future international linear collider (ILC) project. The KEK-ATF damping ring is a $1.3 \mathrm{GeV}$ racetrack type storage ring, which has been able to produce the ultra-low emittance beam [1]. The KEKATF has four wigglers. The performance of the wiggler magnet was tested during the early time of the commission of the damping ring [2]. The commission of the wigglers revealed a problem. A large horizontal closed orbit distortion (COD) was observed when the wigglers were turned on, which came from the beam kick due to the field distribution at both end poles of the wiggler magnets. The beam tuning with the wigglers on was very difficult and the ultra-low emittance beam with the wiggler magnets could not be confirmed due to the large horizontal COD. In 2004, the electric loads (ELs) were installed at each coil of the wigglers to compensate the field distribution. The effect of the ELs and the operational condition are described in the next section. The measurement results for both with and without wigglers are also described.

\section{WIGGLER MAGNET HARDWARE AND OPERATION}

The parameters of the wiggler magnet are summarized in Table 1. The parameters were designed for $1.5 \mathrm{GeV}$ operation. The operation current of the wiggler magnet is $600 \mathrm{~A}(0.8 \mathrm{~T})$ for the $1.3 \mathrm{GeV}$ operation. The radiation loss per turn, $\mathrm{U}_{0}$, and the beam energy, $\mathrm{E}$, are described by the following equations,

\footnotetext{
*Corresponding author: takashi.naito@kek.jp(T.Naito)
}

$$
U_{0}^{[\mathrm{keV}]}=88.5 \frac{E_{[\mathrm{GeV}]}^{4}}{\rho_{[\mathrm{m}]}}, E_{[\mathrm{GeV}]}=0.3 B_{[T]} \rho_{[\mathrm{m}]} .
$$

The energy loss per turn with $\sim 600 \mathrm{~A}$ operation at 1.3 $\mathrm{GeV}$ corresponds to $1000 \mathrm{~A}$ operation at $1.5 \mathrm{GeV}$. The half poles at both ends of the magnet had about $8 \%$ higher integrated magnetic field compared to the full poles without correction. The electric loads are connected to each coil of the half pole in parallel. The electric loads independently decrease the current to each coil of the half pole from the main power supply. The electric load is a semiconductor device, which can keep a constant current. The integrated magnetic field as a function of the EL current is shown in Fig. 1. The integrated magnetic field of the half pole with $100 \mathrm{~A}$ of the EL current has the same field of half of the full pole, while the main power supply current is $600 \mathrm{~A}$.

Table 1:Specifications of the wiggler magnet

\begin{tabular}{ll}
\hline Total Length & $2.0 \mathrm{~m}$ \\
One period & $0.4 \mathrm{~m}$ \\
Full gap & $20 \mathrm{~mm}$ \\
B-peak & $1.62 \mathrm{~T}(@ 1000 \mathrm{~A})$ \\
B-eff & $1.40 \mathrm{~T}(@ 1000 \mathrm{~A})$ \\
Current/pole & $20 \mathrm{KA}(@ 1000 \mathrm{Ax} 2)$ \\
Number of poles & 9 (full pole)+2(half pole) \\
\hline
\end{tabular}

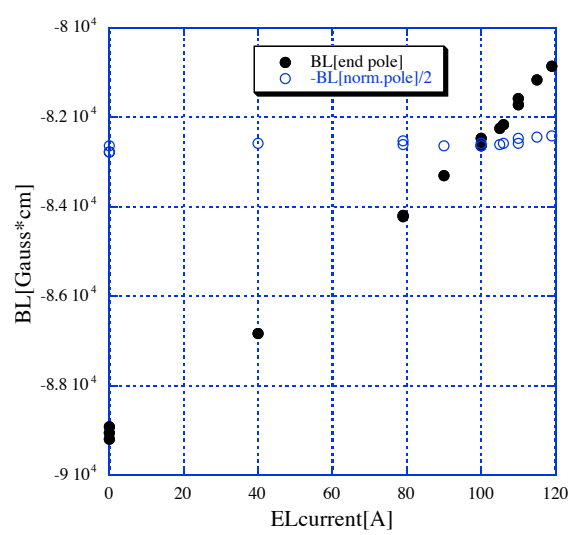

Fig. 1 Integrated magnetic field as a function of the EL current - when the EL current is $100 \mathrm{~A}$, the half pole has the same field of half of the full pole with the main power supply at $600 \mathrm{~A}$. 


\section{Beam Operations with Wigglers}

The beam operations with the wigglers on were studied. The EL current was set to minimize the horizontal COD. The betatron-tune were adjusted with the strength of the focusing magnets such that the tunes were the same wigglers on as wigglers off, $v_{x} \sim 15.18, \quad v_{y} \sim 8.55$, respectively. The edge focusing effect of the wigglers changes the ring optics. The injection matching and chromaticity correction was done to optimize beam storage condition. Fig. 2 shows the field compensation at each wiggler current, the EL current for maximum injection efficiency as a function of the main current of the wigglers, which agrees with the strength of the integrated magnetic field at 600A from Fig.1.

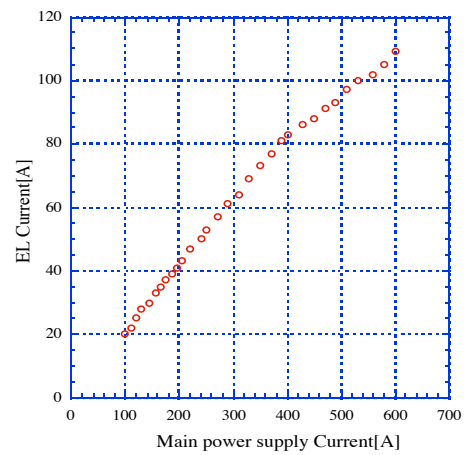

Fig. 2 EL current versus main power supply current at the maximum injection condition

\section{DAMPING TIME AND EMITTANCE SIMULATION AND MEASUREMENT}

The simulation results of SAD [3] are shown in Table 2 for both with and without the wigglers, where $\tau_{x}$ is the horizontal damping time, $\tau_{y}$ is the vertical damping time, $\tau_{z}$ is the longitudinal damping time, $\varepsilon_{x}$ is the horizontal emittance.

Table 2 SAD calculation of the damping times and the horizontal emittance

\begin{tabular}{|l|l|l|}
\hline & $\begin{array}{l}\text { Wigglers off } \\
(1.3 \mathrm{GeV}, 0 \mathrm{~A})\end{array}$ & $\begin{array}{l}\text { Wigglers on } \\
(1.3 \mathrm{GeV}, 600 \mathrm{~A})\end{array}$ \\
\hline$\tau_{\mathrm{x}}$ & $17.5 \mathrm{~ms}$ & $14.2 \mathrm{~ms}$ \\
\hline$\tau_{\mathrm{y}}$ & $28.5 \mathrm{~ms}$ & $21.1 \mathrm{~ms}$ \\
\hline$\tau_{\mathrm{z}}$ & $20.5 \mathrm{~ms}$ & $14.0 \mathrm{~ms}$ \\
\hline $\boldsymbol{\varepsilon}_{\mathrm{x}}$ & $\begin{array}{l}1.03 \times 10^{\wedge}-9 \mathrm{~m} \\
\text { (@zero current) }\end{array}$ & $\begin{array}{c}0.87 \mathrm{x} 10^{\wedge}-9 \mathrm{~m} \\
\text { (@zero current) }\end{array}$ \\
\hline
\end{tabular}

To estimate the $\tau_{x}, \tau_{y}, \varepsilon_{x}$, the beam size was measured by using a Fresnel zone plate monitor (FZP) [4] in the ring and wire scanners (WS) located at the extraction line, downstream of the damping ring. To estimate the $\tau_{z}$, the bunch length was measured by using the streak camera (SC) located at the west arc of the ring. The energy spread was measured by using the screen monitor located at the extraction line, where the dispersion is large. The beam size and the bunch length as a function of time is described by

$$
\sigma^{2}(t) \propto \varepsilon(t)=\varepsilon_{i} e^{-2 t / \tau}+\varepsilon_{e}\left(1-e^{-2 t / \tau}\right),
$$

where $\varepsilon_{i}$ is the initial emittance, $\varepsilon_{e}$ is the equilibrium emittance and $\tau$ is the damping time. The damping time is estimated from the measured beam-size evolution by fitting to the above equation. Fig. 3 shows an example damping-time measurement, namely the square of the horizontal beam size as a function of time in the cases with and without wigglers.

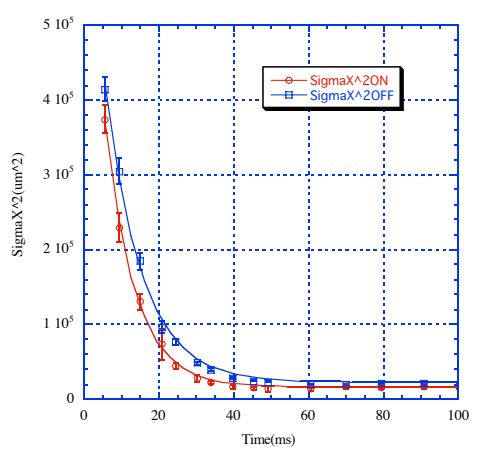

Fig. 3 Beam damping (Horizontal) measured by WS: wiggler on(circle), wiggler off(square)

\begin{tabular}{|l|l|l|}
\hline & $\begin{array}{l}\text { Wigglers off } \\
(1.3 \mathrm{GeV}, 0 \mathrm{~A})\end{array}$ & $\begin{array}{l}\text { Wigglers on } \\
(1.3 \mathrm{GeV}, 600 \mathrm{~A})\end{array}$ \\
\hline $\boldsymbol{\tau}_{\mathrm{x}}$ & $17.2+/-0.43 \mathrm{~ms}(\mathrm{WS})$ & $14.5+/-0.78 \mathrm{~ms}(\mathrm{WS})$ \\
\hline$\tau_{\mathrm{y}}$ & $31.1+/-0.59 \mathrm{~ms}(\mathrm{WS})$ & $22.2+/-0.38 \mathrm{~ms}(\mathrm{WS})$ \\
& $30.9+/-0.9 \mathrm{~ms}(\mathrm{FZP})$ & $20.7+/-0.8 \mathrm{~ms}(\mathrm{FZP})$ \\
\hline$\tau_{\mathrm{z}}$ & $24.5+/-4.5 \mathrm{~ms}(\mathrm{SC})$ & $13.3+/-3.2 \mathrm{~ms}(\mathrm{SC})$ \\
\hline
\end{tabular}

Table 3 Measured damping times

The measured damping times summarized in Table 3 are in good agreement with the SAD calculation. The measurement of the FZP also agrees with the WS measurement.

The growth effect of the intra-beam scattering (IBS) was observed in both the horizontal beam size and the bunch length measurement of the damped beam. The measured energy spread also shows the same behaviour as the bunch length measurement.

The horizontal emittance was estimated from the wire scanner measurement. SAD calculation predicted $\sim 15 \%$ reduction of the horizontal emittance with wigglers at the zero current. Fig. 4 plots the measured emittances with and without wigglers. The intensity dependence was strong due to the IBS effect. It is difficult to compare the zero current emittance. Each measurement point with wigglers showed 10 to $20 \%$ reduction of the horizontal emittance compare to that without wigglers. The line shows the SAD calculation in the case of $0.5 \%$ of $x y-$ coupling and $300 \mathrm{kV}$ acceleration voltage, which almost 
agrees with the horizontal emittance for the case without wigglers. Therefore the measurements without wigglers agree with the SAD calculation and about $15 \%$ emittance reduction was confirmed with the wigglers.

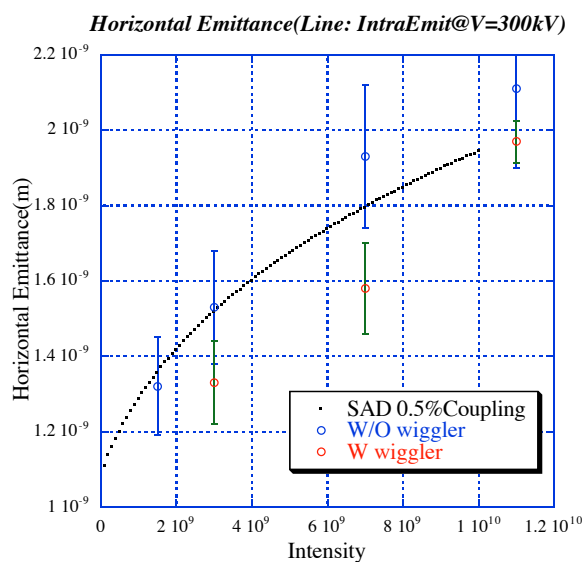

Fig. 4 Horizontal emittance as a function of the intensity measured by WS

The vertical emittance was measured with the laser wire in the damping ring [5]. Fig. 5 shows the emittances as a function of the intensity in the cases with and without wigglers. No emittance growth was seen for the case with wigglers.

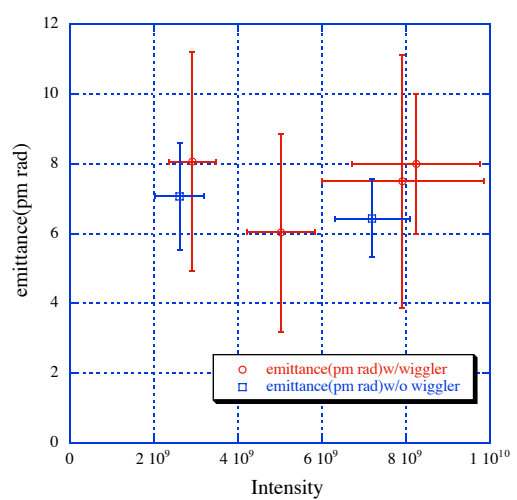

Fig. 5 Vertical emittance as a function of the intensity measured by LW

\section{DYNAMIC APERTURE}

The non-linear effect caused by the wigglers was investigated by measuring the transverse acceptance. The coefficient of beam life $\alpha$ is given by the following equation [6],

$$
\alpha\left[\frac{1}{P a s}\right] \approx \frac{7.2 \times 10^{28}}{P a s m^{2}} \frac{2 \pi r^{2} \sum_{i} Z_{i}^{2}}{\gamma^{2}} \frac{\langle\beta\rangle}{A},
$$

where the transverse acceptance A represents either a physical or a dynamic aperture due to non-linear effect. If the other parameters are constant or known, the transverse acceptance $\mathrm{A}$ is estimated by measuring the beam lifetime. The measurement in the case without wigglers was carried out by F. Zimmermann in 1998 [6]. The measured beam lifetime as a function of the horizontal tune in both cases with and without wigglers is shown in Fig.6. There was no clear difference in the lifetime for either with and without wigglers. Unfortunately, the vacuum levels of the two cases were so different at the two times, so that it is difficult to compare the transverse acceptance.

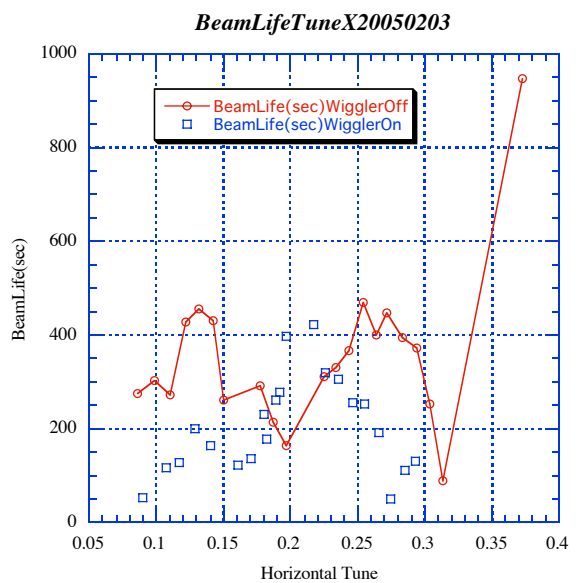

Fig. 6 Beam lifetime measurement as a function of the horizontal tune

\section{SUMMARY}

The operational studies of the wiggler magnets were carried out at KEK-ATF. The field error of the end pole was compensated by using electric loads. The reduction of the damping time was measured by several monitors. The wiggler magnets were confirmed not to cause any measurable deterioration of the vertical emittance and the transverse acceptance.

\section{ACKNOWLEDGEMENT}

We would like to express our thanks to Professors Y.Kamiya and S.Enomoto for their encouragement. We also extend special thanks to ATF group for their help with the accelerator operation.

\section{REFERENCES}

[1] F. Hinode et al., 'Accelerator Test Facility - Design and Study Report', KEK Internal Report 95-4(1995)

[2] T.Naito et al., 'SR Monitor for the ATF Damping', PAC'97, Vancouver, May 1997

[3] $\mathrm{SAD}$ is a computer program complex for accelerator design, http://www-acc-theory.kek.jp/SAD/sad.html

[4] K.Iida et al., 'MEASUREMENT OF AN ELECTRON-BEAM SIZE WITH A BEAM PROFILE MONITOR USING FRESNEL ZONE PLATES' NIM A506:41-49, 2003

[5] Y.Honda et al., 'Achievement of Ultra low Emittance Beam in the Accelerator Test Facility Damping Ring' Physical review letters Vol. 92 No.5 054802

[6] F. Zimmermann et al., 'Transverse Acceptance Studies at the ATF Damping Ring', ATF Internal ATF-98-10 